\title{
A Double-Edged Sword: Neurologic Complications and Mortality in Extracorporeal Membrane Oxygenation Therapy for COVID-19-Related Severe Acute Respiratory Distress Syndrome at a Tertiary Care Center
}

\author{
(D). Masur, (D) C.W. Freeman, and (D) S. Mohan
}

\begin{abstract}
SUMMARY: In this clinical case series, we report our experience to date with neurologic complications of extracorporeal membrane oxygenation therapy for COVID-19 Acute Respiratory Distress Syndrome. We have found an unexpectedly increased rate of complications as demonstrated by neuroimaging compared with meta-analysis data in extracorporeal membrane oxygenation therapy for all Acute Respiratory Distress Syndrome etiologies over the past few decades and compared with the most recent baseline data describing the incidence of neurologic complication in all patients with COVID-19. For our 12patient cohort, there was a rate of intracranial hemorrhage of $41.7 \%$. Representative cases and images of devastating intracranial hemorrhage are presented. We hypothesize that the interplay between hematologic changes inherent to extracorporeal membrane oxygenation and inflammatory and coagulopathic changes that have begun to be elucidated as part of the COVID19 disease process are responsible. Continued analysis of extracorporeal membrane oxygenation therapy in this disease paradigm is warranted.
\end{abstract}

ABBREVIATIONS: ARDS = Acute Respiratory Distress Syndrome; COVID-19 = coronavirus disease 2019; ECMO = extracorporeal membrane oxygenation; MERS = Middle East respiratory syndrome; vv = venovenous

$\mathrm{T}$ he data regarding all aspects of coronavirus disease 2019 (COVID-19) continue to evolve. As the number of patients treated with specific paradigms grows, continuous reevaluation of the outcomes must be undertaken. Extracorporeal membrane oxygenation (ECMO) has been a mainstay of therapy in multiple treatment strategies related to respiratory failure for several decades, including Acute Respiratory Distress Syndrome (ARDS) refractory to conventional therapy. More recently and specifically, ECMO was shown to have mortality reduction benefit in refractory ARDS secondary to the Middle Eastern Respiratory Syndrome coronavirus (MERS-CoV). ${ }^{1}$ As the COVID-19 pandemic has progressed, review of the most recent literature has shown that the ECMO treatment paradigm was thus initiated for severe COVID-19-related ARDS at many institutions. However, as the number of patients

Received May 28, 2020; accepted after revision June 22.

From the Department of Radiology, Hospital of the University of Pennsylvania, Philadelphia, Pennsylvania.

Please address correspondence to Jonathan Masur, MD, Department of Radiology, Hospital of the University of Pennsylvania, 3400 Spruce St., Philadelphia, PA 19104; e-mail: jonmasur@gmail.com

- Indicates open access to non-subscribers at www.ajnr.org

$\equiv$ Indicates article with supplemental on-line table.

http://dx.doi.org/10.3174/ajnr.A6728 treated with various measures including ECMO grows, continuous reevaluation of the outcomes must be undertaken.

Several initial analyses have found that the ECMO paradigm for refractory ARDS in patients with COVID-19 has not shown the same benefit as seen with MERS-CoV. ${ }^{2-4}$ High mortality rates are being reported in these patients compared with those treated with conventional therapy. Although this is undoubtedly multifactorial and small sample sizes have precluded scientifically sound conclusions to date, the early findings are alarming. For example, a recent pooled retrospective analysis of 4 studies in China comprising 567 patients with COVID19 and ARDS of whom 17 were treated with ECMO found a mortality rate of $94 \%$ in the ECMO subgroup compared with $70 \%$ for those treated with conventional therapy alone. ${ }^{4}$

In our group, we are trying to understand the etiopathogenesis and mechanisms of CNS involvement in patients who are positive for COVID-19. Specifically, we are aiming to study the prevalence of neurologic complications and complications of supportive treatments including ECMO. A recent meta-analysis of patients treated with ECMO for respiratory failure of all causes from 1992 to 2015 showed a neurologic complication rate of $7.1 \% ;^{5,6}$ this included a $3.6 \%$ incidence of cerebral hemorrhage and a $1.7 \%$ incidence of stroke. Additionally, a recent observational study from a large academic center reported a rate of intracranial hemorrhage of $4.5 \%$ in 
admitted patients who are positive for COVID-19. ${ }^{7}$ Although there are small case series in the literature regarding ECMO therapy and intracranial hemorrhage in patients with COVID- $19,{ }^{8}$ the data regarding ECMO therapy as it relates to the neurologic sequelae and outcomes of COVID-19 remain sparse. Our larger case series aims to elucidate this relationship.

\section{MATERIALS AND METHODS}

An observational study design was performed with approval from our institutional review board. The number of inpatients admitted to a tertiary health care system between March 1, 2020, and May 19, 2020, with confirmed infection status of "COVID19 positive" was determined by using the "Reports" function of the electronic medical record system (Epic Systems). A list of inpatients with an infection status of "COVID-19 positive" and with neuroimaging of the head performed during the same time period, including CT/CTA and MRI/MRA, was subsequently generated.

The medical records of these participants were examined, and the age, sex, utilization of ECMO, either venovenous (VV) or venoarterial, and duration of ECMO therapy during their admissions were recorded. The rationale for initiation of ECMO in these patients was at the discretion of the treating pulmonologist and according to institutional guidelines. The presence of potentially contributory medical comorbidities in this cohort, including autoimmune conditions, immunosuppression, hypertension, diabetes mellitus, asthma, and structural lung disease, was documented. Additionally, the anticoagulation method used during ECMO as well as the patients' platelet counts at the time of imaging were recorded.

The clinical examination findings prompting neuroimaging in the ECMO cohort were recorded. The images and reports for patients on ECMO with brain imaging were reviewed for critical findings, which we defined as acute or subacute intracranial hemorrhage, acute or subacute infarction, vascular occlusion, findings of infection or inflammation, herniation, hydrocephalus, and evidence of hypoxic-ischemic encephalopathy.

\section{RESULTS}

Between March 1, 2020, and May 19, 2020, we identified 12 patients on VV-ECMO at the time of neuroimaging and none on VA-ECMO. The demographics, comorbidities, anticoagulation therapy, ECMO duration, platelet counts, clinical examination findings prompting neuroimaging, and imaging findings are all presented in the On-line Table.

The most common comorbid conditions in the cohort were hypertension ( 5 of $12 ; 41.7 \%$ ), type 2 diabetes mellitus ( 4 of 12 , $33 \%$ ), and asthma (4 of $12,33 \%$ ). Additional pre-existing conditions that could have predisposed patients to vascular phenomena were present: 1 patient had systemic lupus erythematosus, another had sickle cell trait, and a third had rheumatoid arthritis.

The mean duration of ECMO therapy in our cohort at the time of imaging was 230.3 hours. This is similar to the duration of therapy in the referenced meta-analysis, in which the mean duration of therapy in both the CNS complication and non-CNS complication groups was approximately 258 hours. ${ }^{5}$
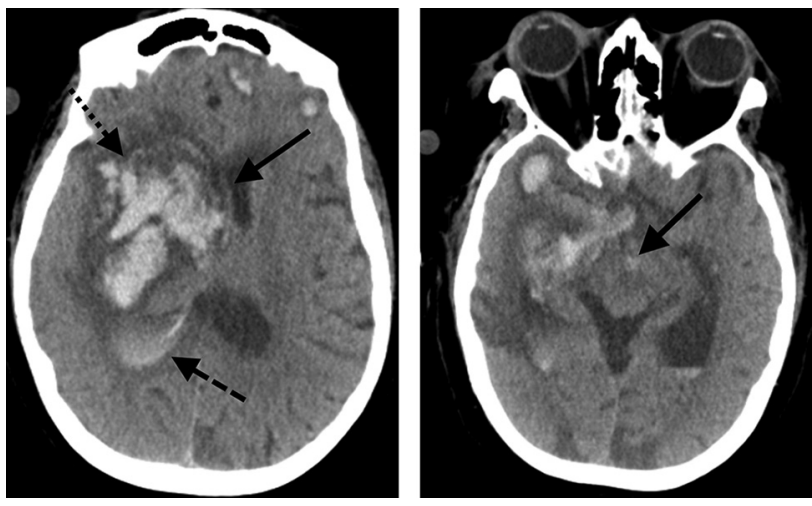

FIG 1. A 65-year-old female patient with a history of rheumatoid arthritis and type 2 diabetes mellitus presented to an outside hospital with fevers and was found to be positive for COVID-19, with pneumonia on chest imaging. The patient's respiratory status declined despite ventilation, prompting transfer and initiation of VV-ECMO. One day after initiation of ECMO, the patient's right pupil was fixed on neurologic examination, prompting emergent head CT. This showed a large mixed-attenuation intraparenchymal hemorrhage centered in the right basal ganglia (dotted arrow) with intraventricular extension (dashed arrow) and right-to-left midline shift or subfalcine herniation (solid arrow). Additional intraparenchymal hemorrhages are seen throughout the supratentorial brain (dotted arrow) as well as ventricular dilation and right uncal herniation. Subarachnoid hemorrhage was also seen, such as in the interpeduncular cistern (solid arrow).
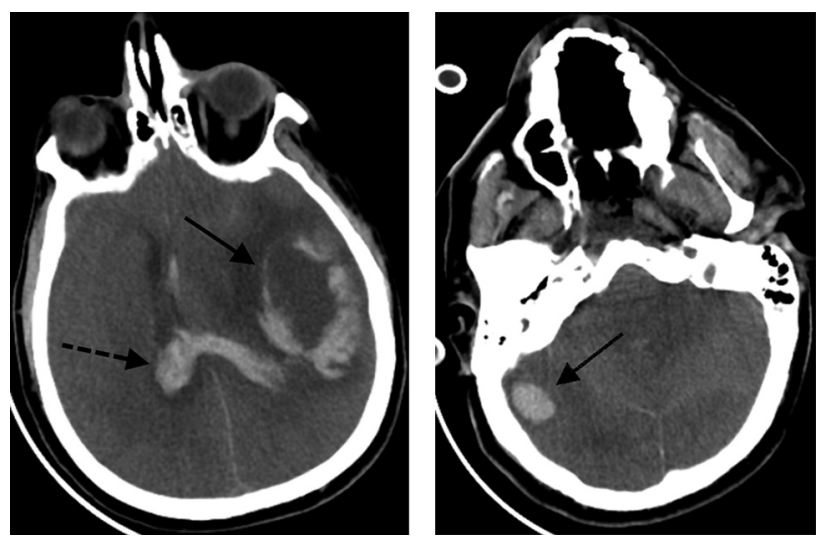

FIG 2. A 60-year-old male patient with a history of asthma and hypertension presented to an outside hospital with fever, dyspnea, and chest imaging demonstrating multifocal pneumonia. The patient tested positive for COVID-19. The patient was intubated, but his condition worsened despite mechanical ventilation, and he was subsequently placed on VV-ECMO. Three days later, the patient demonstrated anisocoria on physical examination, prompting a CT scan of the head. There was diffuse cerebral edema with sulcal and cisternal effacement and multiple intraparenchymal hemorrhages (solid arrows) resulting in left-to-right midline shift, intraventricular extension (dotted arrow), and subfalcine and uncal herniations.

Critical findings meeting our criteria were identified on neuroimaging studies from 6 of the 12 (50\%) participants on ECMO. The most common critical findings were intracranial hemorrhage ( 5 of $12 ; 41.7 \%$ ) with associated herniation in 4 of 5 patients (Figs 1 and 2). All 5 patients had subarachnoid hemorrhage, 4 of 5 had intraparenchymal hemorrhage, and 3 of 5 had intraventricular 
hemorrhage. Evidence of ischemic infarctions were seen in 2 of 6 , both with concomitant intracranial hemorrhage. Three patients had findings of hydrocephalus. All 4 patients with parenchymal hemorrhage and herniation seen on neuroimaging died from the complications. The fifth patient had isolated small-volume subarachnoid hemorrhage that subsequently resolved, but this patient died from ongoing respiratory complications. The sixth patient had findings suggestive of communicating hydrocephalus without a clear cause. One of the 12 patients had vascular imaging with CTA of the head and neck, which did show multiple intracranial large-vessel occlusions; this patient also had multicompartmental intracranial hemorrhages.

\section{DISCUSSION}

Our analysis of the subset of patients with COVID-19-related ARDS treated with ECMO at our institution demonstrates an unexpectedly increased rate (50\%) of devastating neurologic events, specifically intracranial hemorrhage (41.7\%), as demonstrated by emergently performed neuroimaging. Comparatively, a recent meta-analysis of patients treated with ECMO for respiratory failure from 1992 to 2015 showed a neurologic complication rate of $7.1 \%$, including an incidence of cerebral hemorrhage of $3.6 \%{ }^{5}$ Moreover, a recent similarly designed cohort study of 246 consecutive patients who were positive for COVID-19 with neuroimaging at a large tertiary care center showed an incidence of intracranial hemorrhage of $4.5 \%{ }^{7}$ Although the statistical significance of this difference cannot be definitely determined at this time, the results of our analysis do show a trend toward a significantly increased rate of devastating neurologic events manifested on neuroimaging.

Despite the mortality benefit of ECMO demonstrated in patients with MERS-CoV ARDS, we hypothesize that the interplay between the novel COVID-19-related coagulopathy, preexisting conditions, and the hematologic alterations inherent to continuous VV-ECMO could be responsible for the increased risk of neurologic complications in this cohort. This is in keeping with similar mechanisms suggested in the most recent literature. $^{9}$

The main limitation of our analysis is the small sample size to date of patients at our institution treated with ECMO for COVID-19-related ARDS. Given the scope of the coronavirus pandemic and international expert predictions that outbreaks could be experienced for at least the next couple of years, an accelerated prospective clinical trial of the risk versus benefit of ECMO therapy in patients with COVID-19-related ARDS should be considered as feasible. Regardless, continuous retrospective analysis of the available data regarding all aspects of this treatment paradigm is warranted, such that future pooled analyses could add to our work and assess statistical significance of neurologic complications.

\section{CONCLUSIONS}

The evidence continues to mount that ECMO should be used with extreme caution in patients with COVID-19-related ARDS. Very close neurologic monitoring and a low threshold for neuroimaging in this cohort should be used because our findings show a concerning trend toward an increased rate of neurologic complications.

Disclosures: Colbey Freeman—UNRELATED: Grants/Grants Pending: RSNA, NVIDIA, Comments: RSNA resident grant 2020-2021 to institution, NVIDIA GPU grant 2019 provided to me.* Suyash Mohan—UNRELATED: Consultancy: Northwest Biotherapeutics; Grants/Grants Pending: Novocure, Galileo.* *Money paid to the institution.

\section{REFERENCES}

1. Alshahrani MS, Sindi A, Alshamsi F, et al. Extracorporeal membrane oxygenation for severe Middle East respiratory syndrome coronavirus. Ann Intensive Care 2018;8:3 CrossRef Medline

2. Sultan I, Habertheuer A, Usman AA, et al. The role of extracorporeal life support for patients with COVID-19: preliminary results from a statewide experience. J Card Surg 2020;5:1410-13 CrossRef Medline

3. Jacobs JP, Stammers AH, St Louis J, et al. Extracorporeal membrane oxygenation in the treatment of severe pulmonary and cardiac compromise in COVID-19: experience with 32 patients. ASAIO J 2020 2020;66:722-30 CrossRef Medline

4. Henry BM, Lippi G. Poor survival with extracorporeal membrane oxygenation in acute respiratory distress syndrome (ARDS) due to coronavirus disease 2019 (COVID-19): pooled analysis of early reports. J Crit Care 2020;58:27-28 CrossRef Medline

5. Lorusso R, Gelsomino S, Parise O, et al. Neurologic injury in adults supported with veno-venous extracorporeal membrane oxygenation for respiratory failure: findings from the Extracorporeal Life Support Organization Database. Crit Care Med 2017;45:1389-97 CrossRef Medline

6. Peek GJ, Mugford M, Tiruvoipati R, et al. Efficacy and economic assessment of conventional ventilatory support versus extracorporeal membrane oxygenation for severe adult respiratory failure (CESAR): a multicentre randomised controlled trial. Lancet 2009;374:1351-63 CrossRef Medline

7. Radmanesh A, Raz E, Zan E, et al. Brain imaging findings in COVID-19: a single academic center experience in the epicenter of disease in the United States. AJNR Am J Neuroradiol 2020;41:117983 CrossRef Medline

8. Heman-Ackah SM, Su S, Spadola M, et al. Neurologically devastating intraparenchymal hemorrhage in COVID-19 patients on extracorporeal membrane oxygenation: a case series. Neurosurgery 2020;87: E147-51 CrossRef Medline

9. Kowalewski M, Fina D, Słomka A, et al. COVID-19 and ECMO: the interplay between coagulation and inflammation-a narrative review. Crit Care 2020;24:205 CrossRef Medline 